\title{
Necrotising fasciitis following a supracondylar fracture and an open radius fracture in a child
}

\author{
Firth GB ${ }^{1}$, Oetzmann von Sochaczewski C², Ramguthy $Y^{3}$, Khalfallah $A^{4}$ \\ ${ }^{1}$ MBBCh, FCS(Orth), MMed(Orth); Department of Orthopaedic Surgery, University of the Witwatersrand, Chris Hani Baragwanath Academic Hospital, \\ Johannesburg, South Africa \\ ${ }^{2}$ MD, Department of Paediatric Surgery, University of the Witwatersrand, Chris Hani Baragwanath Academic Hospital, Johannesburg, South Africa \\ ${ }_{3}$ MBChB, FCS(Orth); Department of Orthopaedic Surgery, University of the Witwatersrand, Chris Hani Baragwanath Academic Hospital, Johannesburg, \\ South Africa \\ ${ }^{4} \mathrm{MD}$, Department of Orthopaedic Surgery, University of the Witwatersrand, Chris Hani Baragwanath Academic Hospital, Johannesburg, South Africa
}

Corresponding author: Dr GB Firth, University of the Witwatersrand, Chis Hani Baragwanath Academic Hospital, Department of Orthopaedic Surgery, Johannesburg, South Africa; fax: +27117172538; tel: +27826633299; email: greg.firth@gmail.com

\begin{abstract}
Background: Necrotising fasciitis is a rare, rapidly progressing soft-tissue infection with a high mortality rate. Historically, necrotising fasciitis has been associated with penetrating injuries, and more recently with immunocompromise and severe comorbidities. This case report highlights the association of necrotising fasciitis in a child with an open distal radius fracture and a supracondylar fracture.

Method and results (case report): A 10-year-old boy was admitted 24 hours after falling from a tree with a Gustilo and Anderson grade II distal radius fracture and a Gartland grade III supracondylar humerus fracture. The wound was debrided and the fractures reduced and stabilised with Kirschner wires. Within 48 hours of admission he developed a necrotising fasciitis that extended onto the chest and eventually resulted in a shoulder disarticulation. The tissue defects were covered with flaps and skin grafts and the patient was discharged home.

Conclusions: This case highlights the importance of having an early and high index of suspicion for necrotising fasciitis in a child with an open contaminated fracture and delay to both antibacterial chemotherapy and surgical debridement. Tissue trauma due to open fractures may obscure the early skin signs of necrotising fasciitis as well as laboratory risk factors. In the South African context, urgent administration of cephazolin and surgical exploration must be done to prevent the devastating complication of necrotising fasciitis.
\end{abstract}

Level of evidence: Level 5

Key words: necrotising fasciitis, open radius fracture, child, shoulder disarticulation

Citation: Firth GB, Oetzmann von Sochaczewski C, Ramguthy Y, Khalfallah A. Necrotising fasciitis following a supracondylar fracture and an open radius fracture in a child. SA Orthop J 2018;17(2):49-53. http://dx.doi.org/10.17159/2309-8309/2018/v17n2a9

Editor: Dr LC Marais, University of KwaZulu-Natal
Received: July 2017
Accepted: November 2017
Published: May 2018

Copyright: (0) 2018 Firth GB, et al. This is an open-access article distributed under the terms of the Creative Commons Attribution Licence, which permits unrestricted use, distribution and reproduction in any medium, provided the original author and source are credited.

Funding: No financial support was received towards this manuscript.

Conflict of interest: All authors declare they have no conflicts of interest with respect to the writing of this article. 


\section{Introduction}

Historically, penetrating trauma has commonly been described to precede necrotising fasciitis. This has made the condition synonymous with war field injuries. ${ }^{1}$ Its incidence is low in children and diagnosis remains a challenge. ${ }^{2}$ Despite maximum care, the mortality of necrotising fasciitis is as high as $40 \%$, attributed to the failure to recognise the disease early. ${ }^{1}$ Previous non-orthopaedic surgery and minor trauma has been described as the initiating event for necrotising fasciitis in children. ${ }^{3,4} \mathrm{~A}$ recent study reported four cases of necrotising fasciitis following external fixation device implantation for deformity correction or limb lengthening. ${ }^{5} \mathrm{~A}$ toddler with a closed Gartland I supracondylar humerus fracture had necrotising fasciitis attributed to the pressure necrosis secondary to a fibreglass cast. 6 The second paediatric orthopaedic case complicated by necrotising fasciitis, had an open reduction and internal fixation for a closed ankle fracture. ${ }^{7}$ We present a unique case of necrotising fasciitis in a child with an open fracture of the distal radius and an ipsilateral supracondylar humerus fracture (floating elbow). This case highlights the diagnostic challenges posed by this open fracture combination in a South African context which delayed the diagnosis of necrotising fasciitis and resulted in a poor outcome.

\section{Case report}

A 10-year-old boy was admitted to a tertiary care hospital 24 hours after he fell from a tree. He was delayed in getting to the hospital as he first went to two local clinics before arrival in the emergency department. He had no medical comorbidities, his immunisation schedule was up to date, and he had been tested negative for human immune-deficiency virus (HIV). He presented with a left (non-dominant) open distal radius fracture (Gustilo and Anderson grade II) (Figure 1A) and an ipsilateral closed supracondylar humerus fracture (Gartland III) (Figure 1B). The laceration was transverse over the volar aspect of the distal forearm towards the ulnar side, just proximal to the wrist, about $4 \mathrm{~cm}$ long. The wound was contaminated. His radial pulse was not present on the left forearm at presentation, but his fingers were perfusing adequately with a capillary refill time of less than three seconds. He had no neurological deficit at presentation and the injury was isolated to his left upper limb.

The patient received anti-tetanus toxoid vaccine as a booster. In the emergency department a prophylactic dose of intravenous ampicillin was given. After a washout of the distal forearm laceration with normal saline, his fractures were initially stabilised with a backslab. He was taken to the operating room six hours after admission (30 hours after the injury) (Figure 2). At the time of initial surgery, the wound at the distal radius was debrided and both fractures were reduced and internally stabilised with Kirschner wires (K-wires) (Figures 1C, D) and an above-elbow backslab. Prophylactic cephazolin was given perioperatively (three doses). The radial pulse continued to be absent after the fractures were reduced, but his fingers were well perfused (capillary refill time less than three seconds) and it was decided not to explore the radial artery deficiency but to manage it expectantly. On the first post-operative day, the patient was walking around the ward, but complained about pain in his left forearm in keeping with his injury - the pain was not exacerbated by dorsiflexion of the fingers and responded well to analgesia and elevation. The capillary refill time was less than three seconds, but flexion of the little and ring fingers was noted accompanied by a diminished sensation in the ulnar nerve distribution towards the end of the first post-operative day. This ulnar nerve palsy was thought to be due to an iatrogenic injury from the medial distal humerus K-wire insertion. As a result, it was planned to
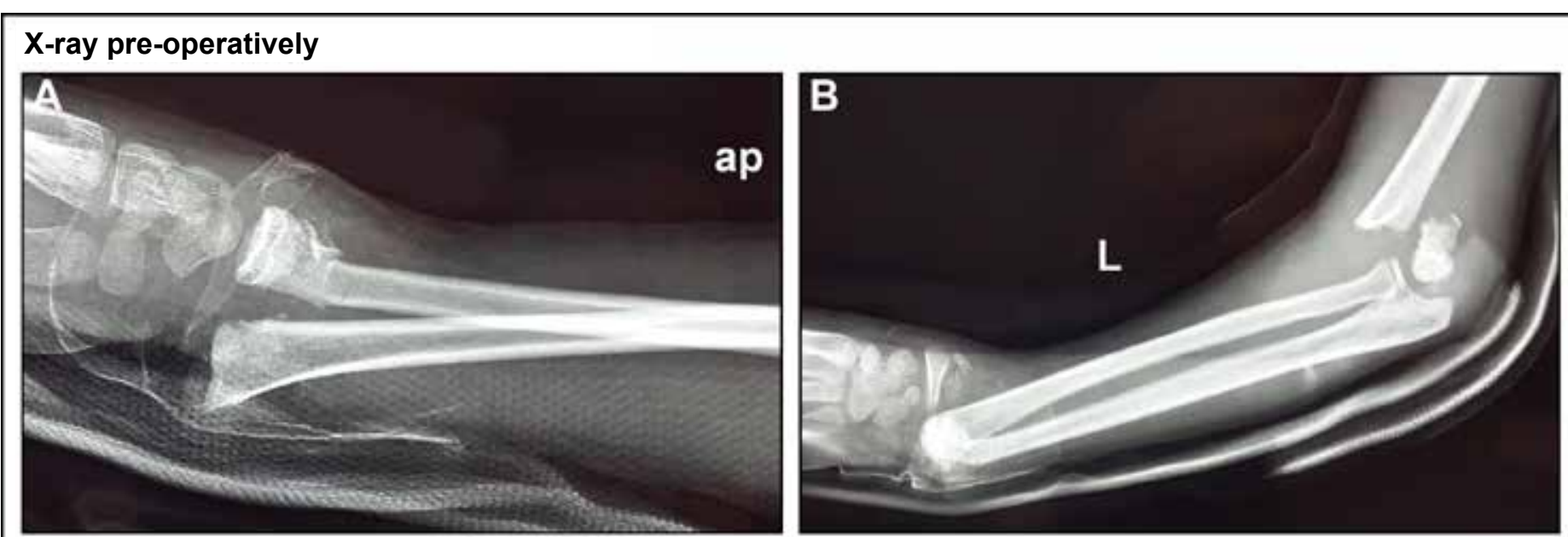

\section{X-ray post-operatively}

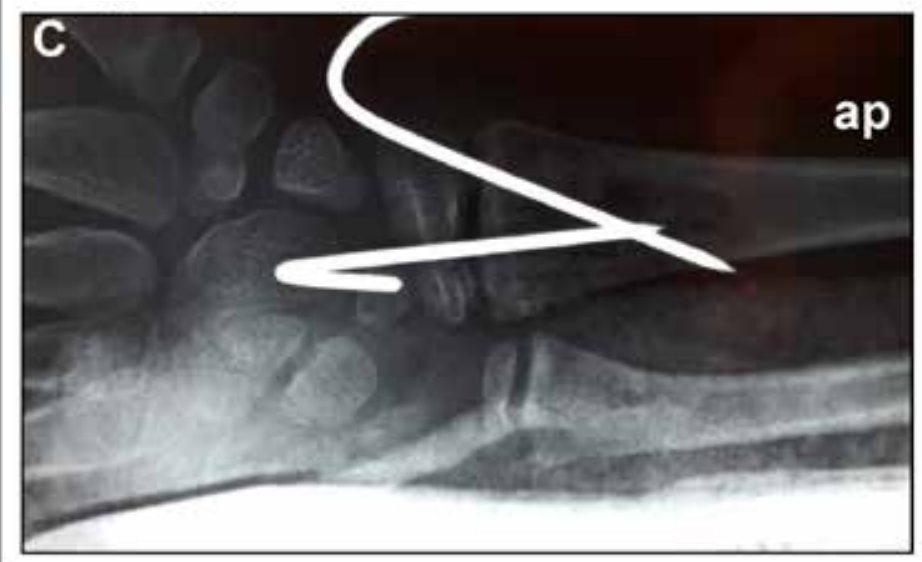

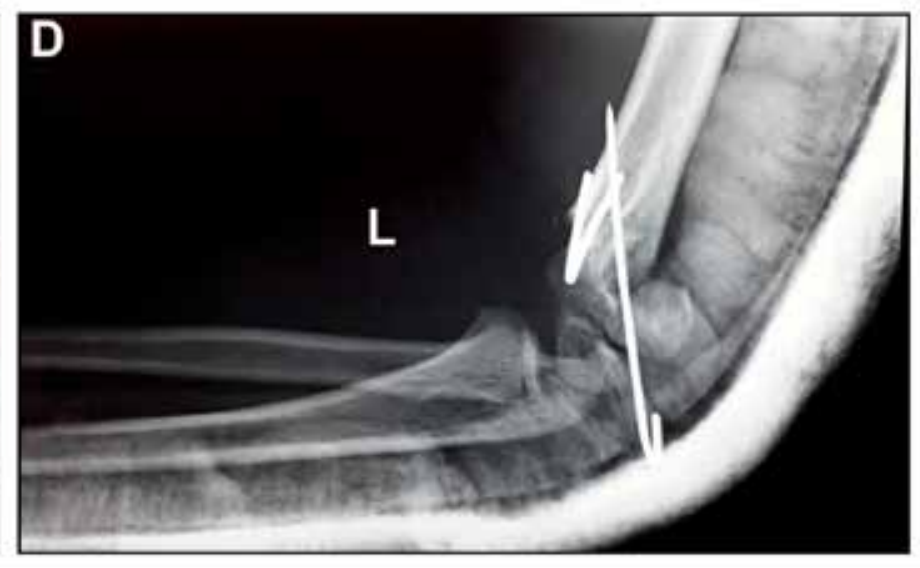

Figure 1. Pre- and post-operative $X$-rays of the fracture 


\begin{tabular}{|c|c|}
\hline \multicolumn{2}{|l|}{ hours } \\
\hline 07 & Initial injury: Patient fell from tree \\
\hline $24-$ & Admission to tertiary hospital \\
\hline $30-1$ & First surgery: Debridement \& ORIF with K-wires \\
\hline $48-$ & \\
\hline $72-$ & \\
\hline $78-$ & Second surgery: Above-elbow amputation \\
\hline 96 & \\
\hline 108 & Third surgery: Shoulder dislocation \& debridement \\
\hline
\end{tabular}

Figure 2. Time line

remove the medial distal humeral wire and replace it with a second lateral wire the following day during a second surgery (48 hours after the initial surgery) that was planned for debridement of wounds. The patient was still walking around the ward on the second post- operative day, but his pain had intensified overnight, and his arm had become more swollen. The radial pulse was still absent, with normal capillary refill time. The ulnar nerve palsy remained unchanged. The symptoms of pain and swelling increased very rapidly in the morning and did not respond to analgesia before the patient was taken to the operating room 78 hours after the initial injury (Figure 2). As a result of his very rapid deterioration, no further investigations (angiography, magnetic resonance imaging or computed tomography) were performed. By the time the patient reached the operating room, surgical emphysema had extended onto the left chest (Figure $3 A$ ), accompanied by erythema of the left arm with extensive skin necrosis (Figure $3 B$ ). The soft tissue and muscles within the arm were necrotic (Figure 3C). At the second surgery, an above-elbow amputation was performed through the supracondylar fracture. At that time, the muscles of the upper arm still looked viable, so the orthopaedic surgeon on call felt that an extension of the debridement to the chest was not necessary. The patient was then admitted to the paediatric intensive care unit, required inotropic support, was started on empiric antibiotic treatment with a combination of intravenous flucloxacillin, piperacillin/tazobactam and clindamycin. Blood tests were ordered for the first time in this case after the admission to the paediatric intensive care unit, around 80 hours after the initial injury: The C-reactive protein was $308 \mathrm{ng} / \mathrm{L}$. His pro-calcitonin level was markedly raised at $275.30 \mu \mathrm{mol} / \mathrm{L}$. The initial white cell count was $5.65 \times 10^{9} / \mathrm{L}$, haemoglobin was $12.5 \mathrm{~g} / \mathrm{dL}$, platelets were $151 \times 10^{9} / \mathrm{L}$ and sodium was $140 \mathrm{mmol} / \mathrm{L}$ after the second surgery. Acute kidney injury had also developed rapidly with an increased anion gap of $21 \mathrm{mmol} / \mathrm{L}$, a urea of $16.4 \mathrm{mmol} / \mathrm{L}$ and a creatinine of $191 \mu \mathrm{mol} / \mathrm{I}$ (AKIN 2). Blood cultures grew Enterobacter cloacae which was resistant to ampicillin and amoxicillin-clavulanic acid but sensitive to ertapenem and cefepime. Enterobacter faecium was also grown and sensitive to ampicillin. Appropriate antibiotic cover was
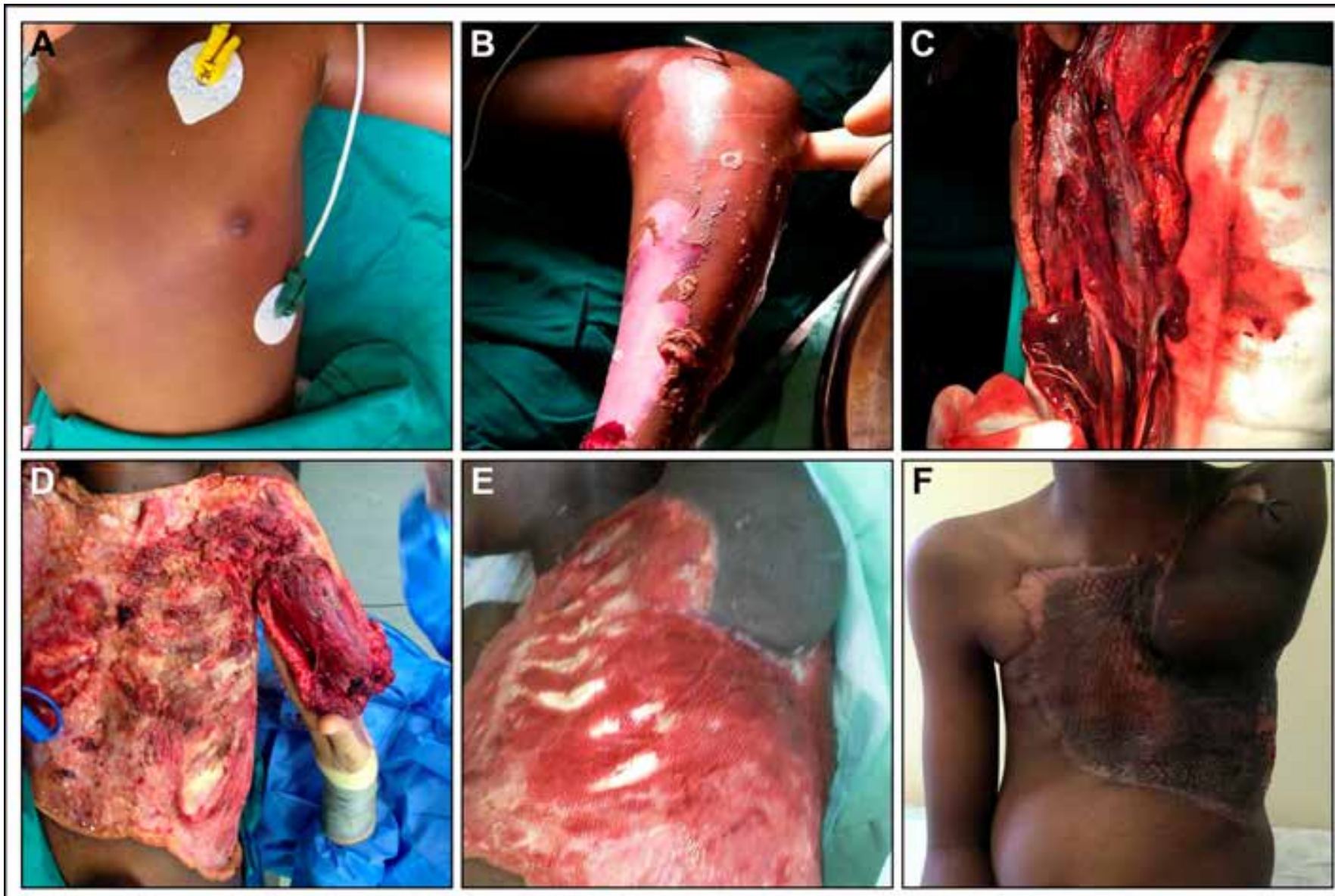

Figure 3. Rapid development of necrotising fasciitis resulting in shoulder disarticulation; flaps and skin grafts cover the tissue defects 
started. The histology at the second surgery showed myonecrosis and extensive acute suppurative inflammation and numerous mixed bacterial colonies. Methicillin-sensitive Staphylococcus aureus was subsequently identified on tissue cultures. A further deterioration on the third post-operative day in terms of his haemodynamic status, further deterioration in his renal function and elevated inflammatory markers despite aggressive medical care at the paediatric intensive care unit, required a third surgery around 108 hours after the initial injury (Figure 2), with extensive debridement of the chest wall including the ribs and sternal surface (Figure 3D). A shoulder disarticulation was conducted as part of the extensive debridement during this third surgery in order to obtain source control. Hyperbaric oxygen was not available at the hospital and thus not used during the course of his treatment. After the third surgery he started to recover slowly despite numerous complications. During his time in the intensive care unit he grew numerous organisms on his blood cultures. These organisms included methicillin-sensitive Staphylococcus aureus, Proteus mirabilis (sensitive to amoxycillin-clavulanic acid), Klebsiella pneumoniae (only sensitive to ertapenem, imipenem and meropenem) and Sphingobacterium species. Despite several complications, including a disseminated intravascular coagulopathy, acute renal failure and a ventilator-associated pneumonia, the wound healed with the assistance of negative pressure vacuum dressings (Figure $3 E$ ) and was eventually covered with local skin flaps and skin grafts (Figure 3F). The patient was discharged home. The renal function recovered to normal levels.

\section{Discussion}

The diagnosis of necrotising fasciitis remains a challenge as skin changes vary widely on presentation. ${ }^{2}$ In a retrospective case series of 39 paediatric cases of necrotising fasciitis, the majority had localised pain, indurated skin, erythema, local warmth and splinting of the affected body region. , $, 8,9$ While the classic hallmarks of skin signs of necrotising fasciitis (bullae and skin necrosis) were highly prevalent in the former, ${ }^{3}$ they were absent in the latter.8,9 The absence of these hallmark signs at presentation was noted to be the most relevant factor in missing the diagnosis., ${ }^{2,7}$ The subtle skin changes indicative of an infectious process have been described to be useful to raise diagnostic suspicion for necrotising fasciitis in non-orthopaedic surgery. ${ }^{10}$ In our case the early skin changes could be attributed both to the initial and surgical tissue trauma and therefore be perceived as normal until red flags appear. One of them is pain out of proportion: the patient experiences pain the intensity of which exceeds the amount of pain that one would expect from the skin signs present. ${ }^{11}$ When it appeared, conjointly with the increased swelling of the arm, the necessity for an urgent second surgery became obvious.

As the initial clinical findings were inconclusive in our case until the red flag of pain out of proportion appeared, imaging might have been helpful to aid the diagnosis: The World Society of Emergency Surgery's guidelines mention computed tomography as well as magnetic resonance imaging as a weak recommendation, ${ }^{12}$ but due to the rapid progression overnight, a further validation of the diagnosis would have further delayed the surgical source control. Early diagnosis is the key to halt disease progression and limit its extension to previously unaffected body parts.,12 The presence of subcutaneous gas on plain X-rays is often described to be of diagnostic value, but due to its insensitivity and occurrence in only a minority of patients, plain X-rays were not considered useful.,2,12,13 Based on the rapid progression of necrotising fasciitis and the timeconsuming nature of imaging, surgical exploration remains the gold standard for both diagnosis and treatment of necrotising fasciitis. ${ }^{12-14}$

Besides imaging modalities, laboratory markers have also been shown to assist in the diagnosis of necrotising fasciitis. The Laboratory Risk Indicator for Necrotising Fasciitis (LRINEC) has been described to aid diagnosis with a positive predictive value of $92 \%$ for the cut- off, using the routine laboratory parameters C-reactive protein, white cell count, haemoglobin, sodium, creatinine, and blood glucose. ${ }^{15}$ However, this score has not been validated for paediatric patients ${ }^{7}$ and only one report describes its successful use in a paediatric patient. ${ }^{16}$ Hence, the LRINEC in a paediatric case-control study of 20 cases of necrotising fasciitis and 20 controls diagnosed with non-necrotising soft tissue infections was found to be inferior to a modification that takes only sodium $<135 \mathrm{mmol} / \mathrm{L}$ and $\mathrm{CRP}>20 \mathrm{mg} / \mathrm{L}$ into account. ${ }^{17}$ In our case, we conducted the first blood analysis after the second surgery on admission to the paediatric intensive care unit, which was after the initially missed diagnosis of necrotising fasciitis had already been established intra-operatively. The applicability of a score that strongly relies on levels of C-reactive protein remains uncertain in post-operative patients and patients with fractures. Fractures cause elevations of C-reactive protein levels. ${ }^{18,19}$ Moreover, the levels of the C-reactive protein experience a steep rise following surgery ${ }^{17-20}$ that renders them unhelpful as a post-operative diagnostic score.

In this case report the diagnosis of necrotising fasciitis was missed, as is the case in almost $75 \%$ of paediatric cases. ${ }^{3}$ Missed diagnosis results in delayed surgical intervention, which correlates with mortality if it exceeds 24 hours. ${ }^{21,22}$ Our case had the second surgery within 24 hours following the onset of symptoms. Necrotising fasciitis has been shown to result in high mortality compared to controls with other serious infectious diseases. ${ }^{23}$ This high mortality has been observed in other serious infectious diseases too, but the rapid onset of symptoms is not rivalled in cases of necrotising fasciitis. ${ }^{24}$ Although the current case survived this devastating disease, despite a delay in diagnosis and intervention, he suffered a severe morbidity, with the loss of his left arm.

On reviewing this case, we noticed several points that might have contributed to the devastating outcome of our patient. The first was poor choice of initial antibiotic: he received ampicillin in the emergency department, which was against our standard operating procedure of cephazolin based on the guidelines of prophylactic antibacterial chemotherapy in open fractures. ${ }^{25}$ The inadequate antibacterial cover might have given the causative organisms the chance to reach deeper structures. Moreover, the 'Eagle effect' has been described for penicillins: a time delay in administration of penicillins reduced their bactericidal effect $^{1}$ and may have also contributed to the initiation of necrotising fasciitis in our patient as 24 hours had already passed from the initial injury and wound contamination. Initial surgical debridement within six hours after the injury has been considered the best treatment of open paediatric fractures, but has been challenged by a systematic review with meta-analysis, which found no difference between surgical debridement within six hours and after 24 hours. ${ }^{26}$ However, immediate antibacterial chemotherapy is regarded as key to successful treatment of open paediatric fractures, ${ }^{27}$ especially when they are managed non-operatively. ${ }^{28}$ Our case presented to our hospital 24 hours after the initial injury and after being seen at two smaller clinics, neither of which initiated antibacterial chemotherapy.

The causative pathogens define the classification of necrotising fasciitis: ${ }^{29}$ a synergism of several pathogens is described to cause polymicrobial type I necrotising fasciitis that is associated with immunocompromise in children ${ }^{29}$ as well as adults, and often has no preceding trauma but a history of comorbidities. ${ }^{13,14}$ In contrast, monomicrobial, usually Gram-positive bacteria, type II necrotising fasciitis tends to occur in otherwise healthy individuals, usually with a preceding trauma or a skin defect.13,14,29,30 The classification also lists a type III necrotising fasciitis caused by monomicrobial Gramnegative bacteria and a type IV due to fungi. ${ }^{29}$ Our patient had a type II necrotising fasciitis as he had an initiating trauma, and only methicillin-sensitive Staphylococcus aureus was isolated from the initial intra-operative tissue cultures.

The final factor that may have contributed to his poor outcome was the absent radial pulse, thus indicating possible hypoperfusion of the area. His capillary refill time was within normal limits, which prompted us to consider the vascular supply sufficient and therefore to refrain from further diagnostic procedures. 


\section{Conclusions}

This case highlights the importance of having an early and high index of suspicion for necrotising fasciitis in a child with an open contaminated fracture and delay to both antibacterial chemotherapy and surgical debridement. Tissue trauma due to open fractures may obscure the early skin signs of necrotising fasciitis as well as laboratory risk factors. In the South African context urgent administration of cephazolin and surgical exploration must be done to prevent the devastating complication of necrotising fasciitis.

\section{Ethics statement}

Informed consent for publication of the case report including the depicted photos was been obtained from the patient's parents. Approval from the Ethics Committee of the University of Witwatersrand (permission number: M1511111) was granted.

\section{References}

1. Lamb LEM, Sriskandan S, Tan LKK. Bromine, bear-claw scratch fasciotomies, and the Eagle effect: management of group A streptococcal necrotising fasciitis and its association with trauma. Lancet Infect Dis 2015;15:109-21. doi:10.1016/ S1473-3099(14)70922-3

2. Jamal N, Teach SJ. Necrotizing fasciitis. Pediatr Emerg Care 2011;27:1195-99; quiz 1200-1202. doi:10.1097/ PEC.0b013e31823b583c

3. Fustes-Morales A, Gutierrez-Castrellon P, Duran-Mckinster C, et al. Necrotizing fasciitis: report of 39 pediatric cases. Arch Dermatol 2002;138:893-99. doi:10.1001/archderm.138.7.893

4. Moss RL, Musemeche CA, Kosloske AM. Necrotizing fasciitis in children: prompt recognition and aggressive therapy improve survival. J Pediatr Surg 1996;31:1142-46.

5. Jauregui JJ, Bor N, Thakral R, et al. Life- and limb-threatening infections following the use of an external fixator. Bone $J t ~ J$ 2015;97-B:1296-300. doi:10.1302/0301-620X.97B9.35626

6. Delasobera BE, Place R, Howell J, et al. Serious infectious complications related to extremity cast/splint placement in children. J Emerg Med 2011;41:47-50. doi:10.1016/j.jemermed.2010.05.031

7. Tancevski A, Bono K, Willis L, et al. Necrotizing fasciitis in a pediatric orthopedic population. Orthopedics 2013;36:e741-745. doi:10.3928/01477447-20130523-18

8. Zerr DM, Alexander ER, Duchin JS, et al. A case-control study of necrotizing fasciitis during primary varicella. Pediatrics 1999;103:783-90.

9. Hsieh T, Samson LM, Jabbour M, et al. Necrotizing fasciitis in children in eastern Ontario: a case-control study. CMAJ Can Med Assoc J J Assoc Medicale Can 2000;163:393-96.

10. Farrell LD, Karl SR, Davis PK, et al. Postoperative necrotizing fasciitis in children. Pediatrics 1988;82:874-79.

11. Borschitz T, Schlicht S, Siegel E, et al. Improvement of a clinical score for necrotizing fasciitis: 'pain out of proportion' and high CRP levels aid the diagnosis. PLOS ONE 2015;10:e0132775. doi:10.1371/journal.pone.0132775

12. Sartelli M, Malangoni MA, May AK, et al. World Society of Emergency Surgery (WSES) guidelines for management of skin and soft tissue infections. World J Emerg Surg 2014;9:57. doi:10.1186/1749-7922-9-57

13. Sarani B, Strong M, Pascual J, et al. Necrotizing fasciitis: current concepts and review of the literature. J Am Coll Surg 2009;208:27988. doi:10.1016/j.jamcollsurg.2008.10.032

14. Shiroff AM, Herlitz GN, Gracias VH. Necrotizing soft tissue infections. J Intensive Care Med 2012;29:138-44. doi:10.1177/0885066612463680

15. Wong $\mathrm{C}-\mathrm{H}$, Khin L-W, Heng K-S, et al. The LRINEC (Laboratory Risk Indicator for Necrotizing Fasciitis) score: a tool for distinguishing necrotizing fasciitis from other soft tissue infections. Crit Care Med 2004;32:1535-41.

16. Song CT, Hamilton $\mathrm{R}$, Song $\mathrm{C}$, et al. Enabling the diagnosis of necrotising fasciitis without associated skin changes in a paediatric patient with acute lymphoblastic leukaemia: the LRINEC score. J Plast Reconstr Aesthetic Surg JPRAS 2015;68:e23-24. doi:10.1016/j.bjps.2014.08.044

17. Putnam LR, Richards MK, Sandvall BK, et al. Laboratory evaluation for pediatric patients with suspected necrotizing soft tissue infections: A case-control study. J Pediatr Surg 2016;51:1022-25. doi:10.1016/j.jpedsurg.2016.02.076

18. Horst $\mathrm{K}$, Hildebrand F, Pfeifer R, et al. Plate osteosynthesis versus hemiarthroplasty in proximal humerus fractures - Does routine screening of systemic inflammatory biomarkers makes sense? Eur J Med Res 2015;20:5. doi:10.1186/s40001-014-0079-z

19. Neumaier M, Scherer MA. C囚reactive protein levels for early detection of postoperative infection after fracture surgery in 787 patients. Acta Orthop 2008;79:428-32. doi:10.1080/17453670710015355

20. Limpisvasti O, Yandow SM, Raney EM. C-reactive protein response following pediatric orthopaedic surgery. J Pediatr Orthop 2004;24:574-75.

21. Wong $\mathrm{C}-\mathrm{H}$, Chang $\mathrm{H}-\mathrm{C}$, Pasupathy $\mathrm{S}$, et al. Necrotizing fasciitis: clinical presentation, microbiology, and determinants of mortality. $J$ Bone Joint Surg Am 2003;85-A:1454-60.

22. $\vee \mathrm{K}$, Hiremath $B V, \vee$ A I. Necrotising soft tissue infection-risk factors for mortality. J Clin Diagn Res JCDR 2013;7:1662-65. doi:10.7860/ JCDR/2013/5535.3240

23. Light TD, Choi KC, Thomsen TA, et al. Long-term outcomes of patients with necrotizing fasciitis. J Burn Care Res Off Publ Am Burn Assoc 2010;31:93-99. doi:10.1097/BCR.0b013e3181cb8cea

24. Thuny F, Giorgi R, Habachi R, et al. Excess mortality and morbidity in patients surviving infective endocarditis. Am Heart J 2012;164:94101. doi:10.1016/j.ahj.2012.04.003

25. Hauser CJ, Adams CA, Eachempati SR. Prophylactic antibiotic use in open fractures: an evidence-based guideline. Surg Infect 2006;7:379-405. doi:10.1089/sur.2006.7.379

26. Ibrahim T, Riaz M, Hegazy A, et al. Delayed surgical debridement in pediatric open fractures: a systematic review and meta-analysis. $J$ Child Orthop 2014;8:135-41. doi:10.1007/s11832-014-0567-2

27. Godfrey J, Pace JL. Type I open fractures benefit from immediate antibiotic administration but not necessarily immediate surgery. $J$ Pediatr Orthop 2016;36 Suppl 1:S6-10. doi:10.1097/ BPO.0000000000000766

28. Godfrey J, Choi PD, Shabtai L, et al. Management of pediatric type I open fractures in the emergency department or operating room: a multicenter perspective. J Pediatr Orthop Published Online First: 10 March 2017. doi:10.1097/BPO.0000000000000972

29. Morgan MS. Diagnosis and management of necrotising fasciitis: a multiparametric approach. J Hosp Infect 2010;75:249-57. doi:10.1016/j.jhin.2010.01.028

30. Frère $J$, Bidet $P$, Tapiéro $B$, et al. Clinical and microbiological characteristics of invasive group a Streptococcal infections before and after implementation of a universal varicella vaccine program: Table 1. Clin Infect Dis 2016;62:75-77. doi:10.1093/cid/civ793 